\title{
CME Neurology (120487): self-assessment questionnaire
}

\author{
Edited by Hannah Cock, Mark Edwards and Tahseen A Chowdhury
}

SAQs and answers are ONLINE for RCP fellows and collegiate members

\section{Format}

Candidates are asked to choose the best answer from the five possible answers. This best of five format is used in many medical examinations; however, the questions are not intended to be representative of those used in the MRCP(UK) Part 1 or Part 2 Written Examinations.

\section{The answering process}

1 Go to www.rcplondon.ac.uk/SAQ

2 Log on using your usual RCP username and password

3 Select the relevant CME question paper

4 Answer all 10 questions by selecting the best answer from the options provided

5 Once you have answered all the questions, click on Submit

\section{Registering your external CPD credits}

Carrying out this activity allows you to claim two external CPD credits. These will be automatically transferred to your CPD diary, where you can review the activity and claim your points.

1. When should lorazepam $4 \mathrm{mg}$ be given in the case of $\mathrm{a}$ prolonged tonic clonic seizure in an adult?
(a) immediately
(b) 2 min
(c) 5 min
(d) $10 \mathrm{~min}$
(e) never.

2. Regarding first seizure presentations in adults, with which of the following features can neuroimaging be safely deferred to an outpatient pathway?
(a) taking anticoagulants
(b) unclassified tonic clonic seizure
(c) known malignancy
(d) new onset focal neurologic deficit
(e) persistent altered mental status.

3. A 24-year-old man presented to the emergency department with a 5-day history of lower back pain with 'shooting, stabbing' pains radiating into both legs. He required assistance to walk, attributing this to pain and 'heaviness' in the lower limbs. There had been no change in bladder or bowel function. He volunteered that he had experienced 'bad diarrhoea' 2 weeks before these symptoms began. There was no past medical history of note. On examination, evidence of proximal and distal weakness in the upper and lower limbs associated with areflexia was found. While the cranial nerve examination was normal, it was noted that he was tachypnoeac and struggling to complete sentences. Vital signs were normal. Chest auscultation was unremarkable.

\section{What is the most appropriate investigation at this stage?}
(a) arterial blood gas
(b) peak flow measurement
(c) forced vital capacity measurement
(d) chest X-ray
(e) D-dimer.

4. In which of these circumstances is imaging not a prerequisite for LP in immunocompetent adults?
(a) Glasgow Coma Scale (GCS) score of 8
(b) suspected viral meningitis with no signs
(c) status epilepticus
(d) right homonymous hemianopia and dysphasia
(e) suspected cerebral abscess with no signs.

5. A 43-year-old woman developed a severe headache with maximum severity within 1 minute while playing golf. On assessment in the emergency department there were no signs except a Horner's syndrome and neck discomfort. What is the most likely diagnosis?
(a) bacterial meningitis
(b) carotid artery dissection
(c) cerebral venous sinus thrombosis
(d) reversible cerebral vasoconstriction syndrome
(e) subarachnoid haemorrhage.

6. A 32-year-old man presented with 6 months of daily headache following 3 months of increasing episodic disabling headaches which he says he has to 'sleep off'. He had been taking daily ibuprofen and sumatriptan for 
many months. It is suspected that he has medicationoveruse headache complicating migraine. What would be the most appropriate course of action?

(a) Continue analgesics as present but start on an appropriate migraine prophylactic agent.

(b) Reduce ibuprofen to 15 days a month and continue sumatriptan as present.

(c) Restrict ibuprofen and sumatriptan to no more than 10 days a month in total.

(d) Restrict ibuprofen to 15 days a month, sumatriptan to 10 days a month and start migraine prophylaxis.

(e) Stop ibuprofen, restrict sumatriptan to 15 days a month and start migraine prophylaxis.

7. A 38-year-old woman presents to the emergency department with a severe right-sided headache that has been present for at least 8 hours. She describes this as unbearable. On examination her right eye was watering and she was intolerant of fundoscopy. What is the most appropriate initial treatment?
(a) co-codamol
(b) high flow oxygen
(c) oral sumatriptan
(d) topiramate
(e) verapamil.

8. A 24-year-old woman is brought unaccompanied to A\&E after collapsing at a music festival around 50 minutes earlier. She had no warning, woke up when she was being stretchered into an ambulance and is now fully alert and orientated. She had been incontinent of urine, bitten the inside of her mouth and $\mathrm{X}$-rays confirm she sustained a distal radius fracture in the fall. Paramedics report that she was floppy and unresponsive when they first arrived, but then had convulsive movements on and off for 8 minutes before coming round. She admits to having taken a 'legal high' earlier that day.

Which feature is most suggestive of this being a dissociative rather than epileptic event?
(a) duration of the convulsive phase
(b) her full recovery
(c) prior recreational drug use
(d) the distal radius fracture
(e) urinary incontinence.

9. Which of the following in a 45-year-old man with a sudden onset left-sided weakness is the strongest pointer towards a diagnosis of functional weakness?
(a) a normal CT brain scan
(b) ability to walk unaided
(c) brief full power demonstrable
(d) childhood sexual abuse
(e) onset while being arrested.

10. Which of the following represents best practice when first explaining the diagnosis of a functional neurological disorder to a patient?

(a) emphasising the role of stress

(b) giving the diagnosis a name and explaining how the diagnosis was reached

(c) reassuring them there is no disease

(d) recommending direct psychiatric referral

(e) starting antidepressant medication.

\section{CME Haematology SAQ}

Answers to the CME SAQ published in Clinical Medicine in August 2018

$\begin{array}{llllllllll}\text { Q1 } & \text { Q2 } & \text { Q3 } & \text { Q4 } & \text { Q5 } & \text { Q6 } & \text { Q7 } & \text { Q8 } & \text { Q9 } & \text { Q10 } \\ \text { (c) } & \text { (b) } & \text { (c) } & \text { (d) } & \text { (c) } & \text { (d) } & \text { (a) } & \text { (c) } & \text { (c) } & \text { (b) }\end{array}$

